\title{
Case Report: Adrenocortical Carcinoma with a Solitary Fibrous Tumor
}

\author{
Mariélle Mendes Orlandi Tambellini ${ }^{1}$, Ana Carolina Silva e Costa ${ }^{2}$, Roberto Odebrecht Rocha ${ }^{2}$ and Danilo \\ Giffoni de Mello Morais Mata ${ }^{2}$ \\ 1. Department of Medical Oncology, Servidor Municipal de São Paulo Hospital, São Paulo 01532-000, Brazil \\ 2. Department of Medical Oncology, Santa Marcelina Hospital, São Paulo 08452-590, Brazil
}

\begin{abstract}
ACC (adrenocortical carcinoma) is an uncommon and aggressive malignancy with an overall five year survival rate from less than $50 \%$. The majority of cases are accidentally diagnosed by imaging studies done for other reasons, so they are often diagnosed in the later stages. Due to the rarity of both neoplasms, the synchronous presentation and the lack of effective therapeutic options; we report a case concerning about a female patient with adrenal cancer with a low grade solitary fibrous tumor who underwent surgery, adjuvant radiotherapy and chemotherapy with good response.
\end{abstract}

Key words: ACC (adrenocortical carcinoma), solitary fibrous tumor, rare neoplasm, Mitotane.

\section{Introduction}

ACC (adrenocortical carcinoma) is a rare tumor with a poor prognosis [1] characterized by a high risk of recurrence after radical resection.

However, there have been few randomized or controlled studies because of the rarity of the tumors.

Surgical resection is the treatment of choice. It is apparently curative, but more than half of all patients will have a recurrence.

Mitotane is an important drug used alone or in combination with the drug cytotoxic in adjuvant or palliative treatments. It has been associated with cures, long term remissions, regression of metastases and increased survival.

The solitary fibrous tumor is a rare mesenchymal neoplasm characterized by proliferation of spindle cells, usually affecting pleura, but it is often located in unusual locations [2].

The incidence of solitary fibrous tumor with adrenocortical carcinoma is extremely rare; there is no report as yet published on this matter.

Corresponding author: MMO Tambellini, M.D., research field: medical oncology.

\section{Case Report}

A 63-year-old female, presented to emergency due to uncontrolled high blood pressure $(230 \times 110 \mathrm{mmHg})$ associated with headache, tremors, excessive sweating, nausea and vomiting.

Pathological past history included high blood pressure controlled with angiotensin inhibitors and diabetes controlled with sulfonylurea.

Her oncological genetic load included her grandchild with PNET (primitive neuroectodermal tumor) in central nervous system.

The patient had no abdominal mass on a physical exam. There were no other relevant data.

Laboratory including full blood count, serum electrolytes, liver and kidney function tests, cortisol, catecholamine and urine metanephrines were all within normal ranges.

CT (computed tomography) of the abdomen revealed the presence of a large left suprarenal mass measuring $138 \times 92 \mathrm{~mm}$ (Fig. 1), with cystic areas (necrosis) and a left inguinal mass with $64 \times 56 \mathrm{~mm}$ (Fig. 2).

The patient underwent an open adrenalectomy and lymphadenectomy. Histopathological result confirmed 


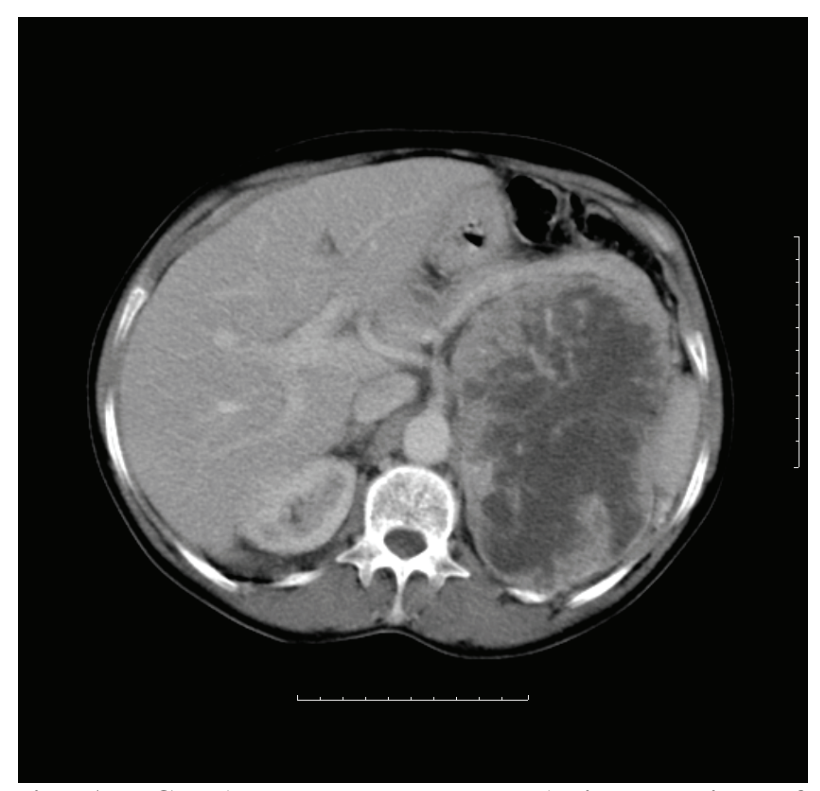

Fig. 1 CT (computed tomography) image with left suprarenal mass.

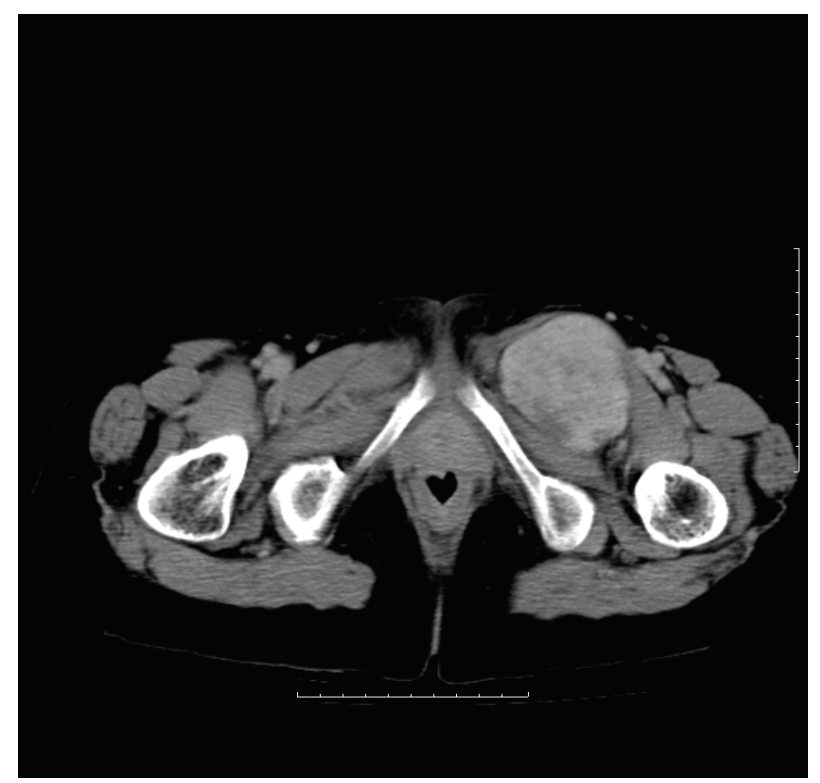

Fig. 2 CT (computed tomography) image with left inguinal mass.

a suprarenal adrenocortical carcinoma and a solitary fibrous tumor, pT3pNxM0 (tumor size: $18 \mathrm{~cm}$, with capsular, sinusoidal and vascular invasion, 2 mitosis/10 high power field and coincident margins).

There was good blood pressure control after surgery.

She came to the Clinical Oncology Department in September 2014 sent by the Urology Department to do an adjuvant treatment evaluation.
We began treatment with $500 \mathrm{mg}$ of Mitotane by mouth once a day with a monthly increasing dose of $500 \mathrm{mg}$ until $2 \mathrm{~g} /$ day (patient maximum dose tolerated).

She underwent radiotherapy with 54 Gy in the left adrenal area.

A six-monthly-radiological follow-up was chosen for the solitary fibrous tumor.

After the second cycle the patient presented Grade 2 lymphopenia, Grade 1 asthenia, dizziness and adrenal insufficiency.

After two years of therapy, she is completely asymptomatic with good Mitotane tolerance with no disease recurrence evident and is experiencing a good quality of life.

\section{Discussion}

Adrenal Carcinoma is an aggressive malignant neoplasm with poor prognosis. It is a rare tumor and represents $0.2 \%$ of all neoplasias. Global incidences are $0.5 \sim 2$ every $1,000,000$ inhabitants per year [3].

It has a bimodal age distribution with higher incidences in children under the age of five and in adults in the fourth and fifth decades of life.

Adrenal carcinoma shows no racial predilection and there is an approximate 4:3 woman to man ratio. Adults generally present with an advanced stage of the disease. Five-year survival rates are discouraging and vary from $16 \%$ to $38 \%$ [4].

Complete surgical resection is the only potentially curative treatment [5] for patients with the localized disease; however, due to a recurrence rate of $50 \sim 70 \%$ after apparent radical surgery, there is a strong rationale for a concomitant systemic treatment [6].

It is known that patients with adrenocortical carcinoma have an extremely poor prognosis when surgical removal of the tumor is not feasible [4].

Mitotane is the only medication approved by US Food and Drug Administration for adrenal carcinoma treatment. It was developed in 1960 as the insecticide DDT (dichlorodiphenyltrichloroethane) [1]. 
Mitotane is an immunosuppressive agent and is described as an adrenal cytotoxic agent, although its exact mechanism of action is unknown, several in-vitro studies have shown that Mitotane suppresses gene transcription of different enzymatic steps of the steroidogenetic pathway [7]. It directly suppresses the adrenal cortex, both with and without cellular destruction. Production of corticosteroids is inhibited and peripheral steroid metabolism is modified, leading to increased excretion of 17-hydroxycorticosteroids and 17-ketosteroids [1, 3].

Mitotane monotherapy is indicated in the management of patients with a low tumor burden and/or more indolent disease while patients whose disease show an aggressive behavior need cytotoxic chemotherapy [8].

Adjuvant Mitotane treatment was associated with some adverse events, which may be considered acceptable, given the disease. Adverse events were manageable, though a temporary reduction of the Mitotane dose was necessary in some patients. Mitotane was not terminated because of adverse events in many of the patients [4].

The most important side effect is adrenal insufficiency, so concomitant glucocorticoid administration is recommended.

Adjuvant radiotherapy is controversial, because of the adrenocortical carcinoma low radiosensitivity.

It is indicated to reduce local recurrence.

Palliative radiotherapy has been considered to reduce symptoms like bone pain, and central nervous system lesion or vena cava syndrome.

However, only limited information on pain reduction, quality-of-life data, and duration of response is given in the published reports [9].

The patient of this case was treated with surgery and adjuvant radiotherapy, because of the positive margins at histopathology and the possibility of local recurrence.

An adjuvant monotherapy was started with $500 \mathrm{mg}$ of Mitotane and progressive increasing dose until
$2 \mathrm{~g} /$ day with low toxicity and no disease evidence until this moment.

The incidence of solitary fibrous tumor with adrenocortical carcinoma is extremely rare.

Solitary fibrous tumor represents a spectrum of mesenchymal tumors. The most common sites of occurrence were abdomen and pleura; these tumors were larger than those occurring in the extremities, head and neck or trunk, but did not demonstrate significant outcome differences.

It is estimated that about $78 \%$ to $88 \%$ are benign and about $12 \%$ to $22 \%$ are malignant [2].

Solitary fibrous tumors are uncommon mesenchymal tumors of the pleural cavity, increasingly recognized at numerous extrathoracic sites [10] and categorized as intermediate biological potential with a low risk of metastasis and relatively indolent course, however, it may undergo malignant transformation.

Complete surgical resection is the treatment of choice. The long-term follow-up is important, because the solitary fibrous tumor can recur locally.

\section{Conclusions}

The incidence of solitary fibrous tumor associated with adrenocortical carcinoma is extremely rare, and there is no report in literature.

Surgical resection is the treatment of choice for both tumors.

Chemotherapy has been used in adjuvant and palliative scenario to control hormonal excess and induce tumor regression.

Adjuvant radiotherapy is ineffective and should be considered only in special cases and palliative treatment.

There has been little progress in the management of the disease, due to the rarity of both tumors, randomized studies are difficult to perform. Besides, new therapeutic strategies are needed.

\section{References}

[1] Luton, J. P., Cerdas, S., Billaud, L., Thomas, G., 
Guilhaume, B., Bertagna, X., et al. 1990. "Adrenocortical Carcinoma and Effect of Mitotane Therapy." The New England Journal of Medicine 322 (17): 1195-201.

[2] Teixeira, U. F., Waechter, F. L., Sampaio, J. A., Pereira-Lima, L. M., and Fontes, P. R. O. 2014. "Tumor Fibroso Solitário Retroperitoneal Volumoso.” Revista da AMRIGS 58 (3): 237-9.

[3] Vilchis-Cárdenas, M. A., López-Verdugo, J. F., Aragón-Tovar, A. R., Torres-Medina, E., Saldaña-Guajardo, M. J., García-Rodríguez, M. A., et al. 2011. "Adrenocortical Carcinoma: A Case Report and Literature Review.” Rev. Mex. Urol. 71 (1): 47-56.

[4] Terzolo, M., Ardito, A., Zaggia, B., Laino, F., Germano, A., De Francia, S., et al. 2012. "Management of Adjuvant Mitotane Therapy Following Resection of Adrenal Cancer." Endocrine 42 (3): 521-5.

[5] Ronchi, C. L., Kroiss, M., Sbiera, S., Deutschbein, T., and Fassnacht, M. 2014. "Current and evolving treatment Options in Adrenocortical Carcinoma: Where Do We Stand and Where Do We Want to Go?.” EJE Prize 2014:
Current and Evolving Concepts in ACC 171: R1-11.

[6] De Francia, S., Ardito, A., Daffara, F., Zaggia, B., Germano, A., Berruti, A., et al. 2012. "Mitotane Treatment for Adrenocortical Carcinoma: An Overview." Minerva Endocrinol. 37 (1): 9-23.

[7] Terzolo, M., Zaggia, B., Allasino, B., and De Francia, S. 2014. "Practical Treatment Using Mitotane for Adrenocortical Carcinoma." Curr. Opin. Endocrinol. Diabetes Obes. 21 (3): 159-65.

[8] Terzolo, M., Daffara, F., Ardito, A., Zaggia, B., Basile, V., Ferrari, L., et al. 2014. "Management of Adrenal Cancer: A 2013 Update.” J. Endocrinol Invest. 37 (3): 207-17.

[9] Polat, B., Fassnacht, M., Pfreundner, L., Guckenberger, M., Bratengeier, K., Johanssen, K., et al. 2009. "Radiotherapy in Adrenocortical Carcinoma." Cancer 115 (13): 2816-23.

[10] Muñoz, E., Prat, A., Adamo, B., Peralta, S., Ramón y Cajal, S., and Valverde, C. 2008. "A Rare Case of Malignant Solitary Fibrous Tumor of the Spinal Cord." Spine (Phila Pa 1976) 33 (12): E397-9. 